\title{
Tolerance Analysis with Multiple Surrogate Models
}

\begin{abstract}
U. BAYRAM* AND E. ACAR
TOBB University of Economics and Technology, Mechanical Engineering Department, Ankara, Turkey

Design and analysis of modern engineering systems are complicated, mostly relying on computational analyses codes and hence often computationally expensive. Meanwhile, improved computer-aided design and numerical simulation methods are used extensively in the design process. Even continuing growth of computing power and speed, computationally cheap and reliable models or simulation techniques are not available, at least as readyto-use computer analysis codes. The necessity of tolerance analysis takes important place at the same time with improved CAD and computer based process planning, because of high quality production requirements, and reduces manufacturing costs. Simulation based design and optimization becomes the only option to meet the specifications, and improves the system reliability. In the last few decades, the use of surrogate models has achieved significant advantages, because they provide fast computation and design investigation as they replace computationally expensive to run computer analyses with cheap to run approximations. Surrogate models also simplify the integration of analyses codes to optimization and reliability assessment studies. In this paper, response surface and Kriging surrogate models are used within a Monte Carlo simulation framework for tolerance analysis. As an illustrative example problem, tolerance analyses of a clutch assembly with nonlinear objection function are used. The effects of the surrogate model parameters (e.g., the use of different regression polynomials) and the number of training points on tolerance analysis are explored.
\end{abstract}

DOI: 10.12693/APhysPolA.128.B-447

PACS: $02.70 .-\mathrm{c}$

\section{Introduction}

As the computational power improves and performance requirements continually tighten, the cost and the specifications of assemblies increased as well. Eventual attention on tolerance analyses is rapidly increasing in engineering field, because higher cost or poor quality performance loses the market share. One of the main factors to determinate the quality of a product and cost is the precision design, which can be accomplished by correct assignment in tolerances. The functionality requirements of an assembly is also important so a robust tolerance analysis is required. The traditional precision design cannot meet the requirement in the digital manufacturing process.

The main objective of this paper is to prove advantage and accuracy of surrogate models on tolerance analysis. Before the explaination of details, fundamentals of surrogate models are described and given a mathematical statement of statistical problem are given. Kriging (KM) $[1,2]$ and response surface methods (RSM) $[3,4]$ are used to compare performance with Monte Carlo simulation (MCS) [5, 6]. This is accomplished by DACE Toolbox [7] and some Matlab code written by using software's embedded library. At the end characteristic of system is discussed with physical system result conducted by Monte Carlo simulations.

\section{Tolerance analysis of mechanism employing a multiple surrogate models}

In this work overrunning clutch assembly, shown in Fig. 1, is considered. This assembly is known as Fortini overrunning clutch assembly [8], and there are many

\footnotetext{
*corresponding author; e-mail: ubayram@etu.edu.tr
}

researchers on this in varied aspects such as Greenwood and Chase [9], Choi et al. [10], Lee et al. [11] and so on.

The problem was examined by Fortini and he formulated the assembly equation as follows:

$$
\begin{gathered}
y=\arccos \left(\left(x_{1}+0.5\left(x_{2}+x_{3}\right)\right)\right. \\
\left./\left(x_{4}-0.5\left(x_{2}+x_{3}\right)\right)\right),
\end{gathered}
$$

where $y$ is the contact angle in terms of the independent component variables, $x_{i}$, which must be functional.

The functionality requirement for this structure is that the contact angle $y$ must lie in the range $0.122 \pm 0.035 \mathrm{rad}$ $(7.0 \pm 2.0 \mathrm{deg})$. Other independent variables are assumed to be Gaussian distribution and when, in order, $x_{i}$ are $55.29,22.86,22.86$, and $101.69 \mathrm{~mm}$, standard deviations are given as 0.1016, 0.01016, 0.01016, $0.2032 \mathrm{~mm}$, respectively.
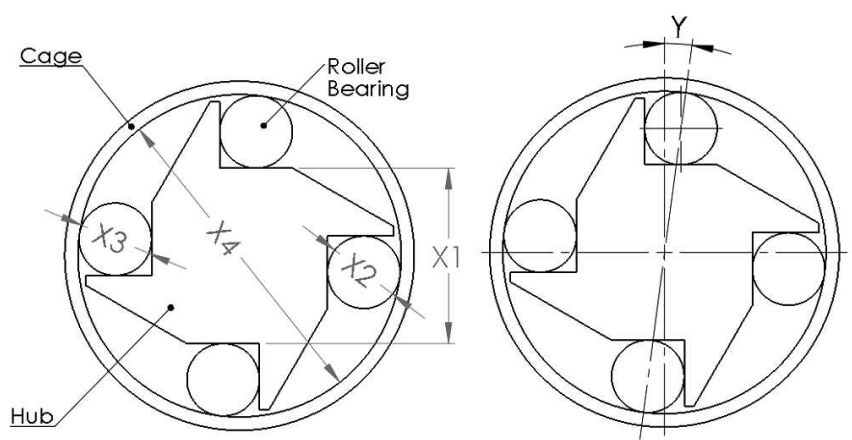

Fig. 1. Fortini's overrunning clutch assembly.

System characteristic is investigated by applying MCS depending on design requirements, and listed in Table I. Between ten thousand and a million normally distributed random numbers were generated by using Matlab embedded function, "icdf". 
TABLE I

Overrunning clutch assembly results using KM.

\begin{tabular}{c|c|c|c|c|c|c}
\hline \hline & \multicolumn{3}{|c|}{40 training points } & \multicolumn{3}{c}{100 training points } \\
\hline outputs of controlled assembly variable & regpoly0 & regpoly1 & regpoly2 & regpoly0 & regpoly1 & regpoly2 \\
\hline mean [radian] & 0.1189 & 0.1305 & 0.1296 & 0.1267 & 0.1299 & 0.1297 \\
standard deviation [radian] & 0.0067 & 0.0244 & 0.0226 & 0.0097 & 0.0229 & 0.0228 \\
skewness & 0.2824 & -0.0764 & -0.6835 & -0.5232 & -0.1469 & -0.6010 \\
kurtosis & 7.3471 & 3.0709 & 3.6900 & 4.9438 & 3.0664 & 3.5702 \\
rejects at upper limits [\%] & 0.01 & 13.45 & 9.56 & 0.19 & 11.19 & 10.2 \\
rejects at lower limits [\%] & 0.08 & 4.04 & 4.37 & 0.12 & 3.6 & 4.33 \\
total rejects [\%] & 0.09 & 17.49 & 13.93 & 0.31 & 14.79 & 14.53
\end{tabular}

\section{TABLE II}

Overrunning clutch assembly results using MCS.

\begin{tabular}{c|c|c|c}
\hline \hline & \multicolumn{3}{|c}{ Number of simulations } \\
\hline outputs of controlled assembly variable & 10,000 & 100,000 & $1,000,000$ \\
mean [radian] & 0.1287 & 0.1293 & 0.1293 \\
standard deviation [radian] & 0.0245 & 0.0238 & 0.0236 \\
skewness & -0.8513 & -0.7185 & -0.7316 \\
kurtosis & 4.6820 & 4.0620 & 4.2006 \\
rejects at upper limits [\%] & 10.0900 & 10.0430 & 9.9619 \\
rejects at lower limits [\%] & 5.4100 & 4.7020 & 4.6510 \\
total rejects [\%] & 15.5000 & 14.7450 & 14.6129
\end{tabular}

TABLE III

Overrunning clutch assembly results using RSM.

\begin{tabular}{|c|c|c|c|c|c|c|c|c|}
\hline & \multicolumn{4}{|c|}{40 training points } & \multicolumn{4}{|c|}{100 training points } \\
\hline $\begin{array}{c}\text { Outputs of controlled } \\
\text { assembly variable }\end{array}$ & Linear & Interactions & Quadratic & $\begin{array}{c}\text { Pure } \\
\text { quadratic }\end{array}$ & Linear & Interactions & Quadratic & $\begin{array}{c}\text { Pure } \\
\text { quadratic }\end{array}$ \\
\hline mean [radian] & 0.1304 & 0.1307 & 0.1296 & 0.1302 & 0.1299 & 0.1300 & 0.1297 & 0.1299 \\
\hline standard deviation [radian] & 0.0245 & 0.0247 & 0.0226 & 0.0235 & 0.0231 & 0.0232 & 0.0228 & 0.0230 \\
\hline skewness & -0.0375 & -0.0234 & -0.6838 & -0.3555 & -0.0368 & -0.1227 & -0.5918 & -0.3793 \\
\hline kurtosis & 2.9850 & 3.0006 & 3.6737 & 3.1807 & 2.9853 & 3.0335 & 3.5087 & 3.2184 \\
\hline rejects at upper limits [\%] & 13.78 & 14.2 & 9.5 & 12.24 & 11.87 & 12 & 10.29 & 11.27 \\
\hline rejects at lower limits [\%] & 3.83 & 3.79 & 4.38 & 4.1 & 3.15 & 3.44 & 4.32 & 3.93 \\
\hline total rejects $[\%]$ & 17.61 & 17.99 & 13.88 & 16.34 & 15.02 & 15.44 & 14.61 & 15.2 \\
\hline
\end{tabular}

MCS results in Table II show that ten thousand samples are convergent according to one million simulations. When accuracy and cost of calculation are considered, simulations with 10,000 samples are assumed to be adequate for this problem.

To perform tolerance analyses of the clutch assembly using surrogate based Monte Carlo simulation, first the Kriging method is applied on this example. The Kriging models are constructed with "dacefit" Matlab function with Gaussian correlation parameter. To see the effect of the number of training points on the accuracy of the Kriging models, 40 and 100 training points are used. The effect of regression model is also examined by creating linear, first and second degree regression polynomial. After the Kriging models are constructed, MCS is used to perform tolerance analysis. 10,000 simulation points are generated for MCS. The results are shown in Table I.

Higher order regression polynomials help to get better approximation according to Table I. Increasing the number of training points used to construct Kriging model from 40 to 100 does not have a significant effect in KM accuracy for higher degree regression polynomials. Comparison of sample based MCS and KM based MCS shows that the performance of KM based MCS with higher regression polynomial is as good as sample based MCS (notice that the difference is less than $2 \%$ ).

The RSM is then applied on this example with the same number of training points, as shown in Table III. While generating RSM models, we take advantage of the "regstats" function of embedded Matlab library to find 
coefficients. As an predictor, " $x \mathscr{2} f x$ " function is used. Different RSM formulations such as linear, quadratic, etc. are also utilized to compare with KM. The results are shown in Table III.

Quadratic regression model at RSM corresponds with regpoly2 at KM. Table III shows that higher order regression polynomial similar to KM should be used in order to increase the accuracy. Increase of the number of training points from 40 to 100 seems not to make more difference in predictions of RSM. The comparison of the results in Table I and Table III shows that the performances of KM with second order trend model and quadratic RSM are close to each other.

\section{Conclusion}

In this paper, response surface and Kriging surrogate models are used within a Monte Carlo simulation framework for tolerance analysis. Tolerance analyses of a clutch assembly with nonlinear objection function is used as an illustrative example problem. The effects of the surrogate model parameters (such as the use of different regression polynomials) and the number of training points on tolerance analysis are explored. From the finding of this study, the following conclusions can be drawn:

- The performances of quadratic RSM and KM with second order regression polynomial were close.

- Comparison of sample based MCS and surrogate based MCS shows that the performance of surrogate based MCS is as good as sample based MCS.

- Increase of the number of training points from 40 to 100 did not increase much the accuracy of surrogate models.

In future work the tolerance analysis may include the use of a more complex objective function with more design variable, and the use of other surrogate model types than RSM and Kriging. Also, the application of the surrogate based tolerance analysis to a complicated 3D tolerance analysis example is a future direction of research.

\section{References}

[1] S. Koziel, D. Echeverría Ciaurri, L. Leifsson, in: Computational Optimization, Methods and Algorithms, Eds.: S. Koziel, Xin-She Yang, Springer, Berlin 2011, p. 33.

[2] T.W. Simpson, Comparison of Response Surface and Kriging Models in the Multidisciplinary Design of an Aerospike Nozzle, Institute for Computer Applications in Science and Engineering, NASA Langley Research Center, Hampton 1998.

[3] R.H. Myers, C.M. Anderson-Cook, Response Surface Methodology: Process and Product Optimization Using Designed Experiments, Wiley, New York 2009.

[4] D.C. Montgomery, Design and Analysis of Experiments, Vol. 7, Wiley, New York 1997.

[5] A.J. Qureshi, J.-Y. Dantan, V. Sabri, P. Beaucaire, N. Gayton, Computer-Aided Des. 44, 132 (2012).

[6] S.D. Nigam, J.U. Turner, Computer-Aided Des. 27, 6 (1995).

[7] S.N. Lophaven, H.B. Nielsen, J. Søndergaard, DACE, a Matlab Kriging Toolbox v. 2.0, 2002.

[8] E.T. Fortini, Dimensioning for Interchangeable Manufacture, Industrial Press, New York 1967.

[9] W.H. Greenwood, K.W. Chase, J. Eng. Industry 112, 382 (1990).

[10] H.-G.R. Choi, E. Salisbury, M.-H. Park, J. Manufact. Sci. Eng. 122, 529 (2000).

[11] S.H. Lee, M.K. Byung, Structural Safety 28, 261 (2006). 